\title{
Can task-switching training enhance executive control functioning in children with attention deficit/-hyperactivity disorder?
}

\author{
Jutta Kray ${ }^{1}$, Julia Karbach ${ }^{1}$, Susann Haenig ${ }^{2}$ and Christine Freitag ${ }^{3}$ \\ 1 Department of Psychology, Saarland University, Saarbruecken, Germany \\ 2 Department of Child and Adolescent Psychiatry and Psychotherapy, Saarland University Hospital, Homburg/Saar, Germany \\ ${ }^{3}$ Department of Child and Adolescent Psychiatry, Psychosomatics and Psychotherapy, Goethe University, Frankfurt/Main, Germany
}

\section{Edited by:}

Torsten Schubert, Ludwig-Maximilians University Munich, Germany

\section{Reviewed by:}

Tilo Strobach, Ludwig-Maximilians University Munich, Germany

Kathrin Finke, Ludwig-Maximilians

University Munich, Germany

\section{*Correspondence:}

Jutta Kray, Department of

Psychology, Saarland University, P.O.

Box 1511 50, D-66041 Saarbruecken,

Germany.

e-mail: j.kray@mx.uni-saarland.de
The key cognitive impairments of children with attention deficit/-hyperactivity disorder (ADHD) include executive control functions such as inhibitory control, task-switching, and working memory (WM). In this training study we examined whether task-switching training leads to improvements in these functions. Twenty children with combined type ADHD and stable methylphenidate medication performed a single-task and a task-switching training in a crossover training design. The children were randomly assigned to one of two groups. One group started with the single-task training and then performed the task-switching training and the other group vice versa. The effectiveness of the task-switching training was measured as performance improvements (relative to the single-task training) on a structurally similar but new switching task and on other executive control tasks measuring inhibitory control and verbal WM as well as on fluid intelligence (reasoning). The children in both groups showed improvements in task-switching, that is, a reduction of switching costs, but not in performing the single-tasks across four training sessions. Moreover, the task-switching training lead to selective enhancements in task-switching performance, that is, the reduction of task-switching costs was found to be larger after task-switching than after single-task training. Similar selective improvements were observed for inhibitory control and verbal WM, but not for reasoning. Results of this study suggest that taskswitching training is an effective cognitive intervention that helps to enhance executive control functioning in children with ADHD.

\section{Keywords: ADHD, task-switching training, executive control, training transfer}

\section{INTRODUCTION}

The main goal of the present study was to determine the range of plasticity in executive control functioning in children with attention deficit/-hyperactivity disorder (ADHD). Executive control can be defined as a set of higher-order cognitive functions that organize and regulate goal-directed behavior including processes of planning, interference control, working memory (WM), taskswitching, and task coordination (e.g., Miyake et al., 2000). Behavioral deficits observed in children with ADHD are characterized by inattention, impulsivity, and hyperactivity (American Psychiatric Association, 1994), and it has been suggested that those deficits are primarily related to executive control impairments, such as inhibitory control and WM (Barkley, 1997; Willcutt et al., 2005).

One experimental task that has frequently been applied in recent years to examine executive control functioning is the taskswitching paradigm (for a recent review; Kiesel et al., 2010). The advantage of this paradigm is that it allows the separation of different components of executive control, such as task-set selection and maintenance, task-set switching, and interference control (Cepeda et al., 2001). In this type of task, the participants are usually instructed to switch between two simple cognitive tasks. For example, the participants are presented ambiguous stimuli, such as a series of digits varying in number and value $(1,3,111,333)$. In one task (task A), they have to decide whether the value of digits is one or three, and in the other task (task B), whether the number of digits is one or three. Performance can be measured in mixedtask blocks, in which the participants have to switch between both tasks A and B on every second trial, and in single-task blocks, in which only one of the tasks (A or B) has to be performed (Kray and Lindenberger, 2000; Kray et al., 2008). This allows the determination of two types of performance costs associated with the switching situation: mixing costs are defined as the difference in mean performance between mixed-task and single-task blocks and are assumed to refer to the ability to maintain two task sets and select between them. Switching costs are defined as the difference in mean performance between switch and non-switch trials within mixed-task blocks and they are assumed to measure the ability to flexibly switch between tasks (cf. Kray and Lindenberger, 2000; Kray et al., 2008). Finally, the efficiency of interference control can be measured by comparing the performance on congruent trials (in which the number and value decisions are not conflicting, i.e., 1, 333) with performance on incongruent trials (in which the number and value decisions are conflicting, i.e., 3, 111), 
that is, interference costs can be defined as the difference in mean performance between incongruent trials and congruent trials.

Cepeda et al. (2000) examined switching and interference costs in ADHD children (6-12 years old), on and off medication, in comparison to children without ADHD that were matched by age and IQ. Results of this study revealed that only ADHD children off medication showed larger switching costs and interference costs than healthy controls but there were no performance differences in these costs between ADHD children on medication and the control children. Moreover, switching costs in ADHD children off medication were only larger on incongruent trials, suggesting that children with ADHD particularly had problems to inhibit irrelevant task information when switching from one task to the other one (Kramer et al., 2001).

Given that children diagnosed with ADHD usually achieve lower academic degrees compared to equally cognitively able children without $\mathrm{ADHD}$, and also have major problems in everyday functioning until adulthood (Rasmussen and Gillberg, 2001), the question of effective treatments, such as cognitive training interventions that help to improve executive control functioning, is of high relevance. One desirable feature of cognitive training interventions is that the training program does not only result in an improvement on the trained task, but that it also transfers to tasks that were not part of the training intervention (Lövdén et al., 2010). To determine the scope of transfer, we distinguish between near and far transfer. Near transfer refers to a generalization of training-related improvements to a new but structurally similar transfer task (e.g., transfer of task-switching training to another switching task, Karbach et al., 2010; Minear and Shah, 2008), while far transfer refers to dissimilar theoretical constructs (e.g., transfer of task-switching training to a WM task; cf. Karbach and Kray, 2009).

In a recent lifespan study, we investigated near and far transfer of task-switching training in children, younger, and older adults with a pretest-training-posttest design (Karbach and Kray, 2009). Pretest and posttest consisted of a cognitive test battery including several tests measuring task-switching (near transfer), interference control, verbal and visual WM, and fluid intelligence (far transfer). Importantly, we included an active control group in this study. Transfer was defined as relative performance improvements at posttest in the treatment group (task-switching training) as compared to the control group (single-task training). Note that both groups performed the identical number and type of A and B tasks, but the control group performed them in separate blocks (singletask training), while the training group switched between both tasks on every second trial (task-switching training). Results indicated that (a) all three age groups showed near transfer effects, that is, a larger reduction of mixing and switching costs from pretest to posttest in the training group than in the control group; (b) near transfer effects were more pronounced in children and older adults than in younger adults; and (c) far transfer effects were observed in all age groups, that is, performance improvements in interference control, verbal and visual WM, and fluid intelligence. The effect sizes for the group of children were between $d^{\prime}=1.2-2.1$ for near transfer and $d^{\prime}=0.5-0.9$ for far transfer of task-switching training. Given these promising effects of the $\operatorname{cog}$ nitive training intervention in healthy children, the specific aim of the present study was to examine whether the training is of similar effectiveness in a group of children with substantial impairments in executive control.

There are a few studies demonstrating that training of executive control in children with ADHD leads to near as well as far transfer effects. Klingberg et al. $(2002,2005)$ used an adaptive training procedure including visuospatial and verbal WM tasks. They found performance improvements not only on the trained visuospatial WM task but also on non-trained tasks assessing visual-spatial memory, fluid intelligence (the Raven's), and interference control. More recently, Shalev et al. (2007) applied an attentional training program in order to improve school performance (e.g., math exercises, reading comprehension) and behavior (parents' self-reports of ADHD symptoms) in ADHD children (6-13 years old). The attentional training included the practice of sustained and selective attention, orienting of attention, and executive attention. The authors found training-related improvements in school performance as well as a reduction of inattention symptoms reported by the parents. Although these far transfer effects are impressive, it should be noted that the authors did not report the improvements on the trained tasks and they did not measure near transfer effects. Kerns et al. (1999) used a similar attentional training including seven ADHD children (7-11 years old) and reported far transfer effects to a number of attentional tasks that were not trained during the intervention.

The main goal of the present study was to determine the transfer scope after task-switching training in ADHD children. Therefore, we investigated near and far transfer effects of this training, similar to a previous study (Karbach and Kray, 2009). For ethical reasons (see also Procedure), we applied a crossover training design so that all $\mathrm{ADHD}$ children performed the cognitive intervention (i.e., the task-switching training) that has already been shown to enhance executive control functioning in healthy young children. However, they received the treatment at different times during the training protocol. That is, after performing the pretest, the children were randomly assigned to one of two groups: group 1 first performed the single-task training followed by posttest 1 and then the task-switching training followed by posttest 2 (see Table 1). Group 2 first performed the task-switching training as well as the first posttest and then the single-task training and the second posttest.

On the basis of previous results showing near and far transfer effects of WM and attentional control training in children with ADHD (Klingberg et al., 2002, 2005; Shalev et al., 2007) as well as near and far transfer effects of task-switching training in healthy young children (Karbach and Kray, 2009), we expected treatment-specific effects in this study. In particular, we predicted a larger reduction of mixing and switching costs after the treatment (task-switching training) than after the single-task training (near transfer) as well as larger improvements in executive control and fluid intelligence measures (far transfer). That is, group 2 should show larger performance improvements from pretest to posttest 1 as compared to group 1, and group 1 should show larger improvements from posttest 1 to posttest 2 than group 2. Given that far transfer effects are usually the smaller the less similar the training and the transfer tasks are, we also expected larger effect sizes for near than for far transfer. 


\section{MATERIALS AND METHODS PARTICIPANTS}

Thirty children were recruited for this study. Ten participants had to be excluded from the analysis because they were not willing to finish the training study $(n=7)$ or went off medication during the study $(n=3)$. Given that ADHD is more common in boys than girls (Froehlich et al., 2007), we included only male children. The final sample consisted of 20 boys that were randomly assigned to one of the two training conditions (group 1: $n=10$, group 2: $n=10)$. Both groups were comparable in terms of age $(p=1.00$; group 1: range $=8.7-12.1$ years; group 2 : range $=7.7-$ 11.6 years $)$ and IQ $(p=0.44)$. The severity of the ADHD-related symptoms was assessed by means of the German parent rating scale FBB-HKS (Döpfner and Görtz-Dorten, 2008). The questionnaire is based on the DSM-IV and ICD-10 criteria for ADHD and hyperkinetic disorders and allows the assessment of behavioral symptoms on the four scales (1) severity of inattention, (2) severity of hyperactivity/impulsivity, (3) generalized inattention problems, and (4) generalized hyperactivity/impulsivity problems. We found no between-group differences on any of the four scales (all $p s>0.53$ ). Means and SD for age, IQ, and parent ratings are provided in Table 2.

All participants were enrolled in mainstream elementary and secondary schools. Prior to the inclusion into the study, they had been diagnosed according to the guidelines of DSM-IV (American Psychiatric Association, 1994) at the Department of Child and Adolescent Psychiatry, Saarland University Hospital, Germany.
The diagnosis was based on a structured interview (K-DIPS, Unnewehr et al., 1998), an intelligence assessment (WISC-IV, Petermann and Petermann, 2007), and standard rating scales (such as the FBB-HKS, Döpfner and Görtz-Dorten, 2008) administered by expert physicians and psychologists.

After being diagnosed, the children had been medicated with methylphenidate. Although individual doses varied as a function of body weight and severity of the symptoms, most of the boys $(n=18)$ were prescribed $10-20 \mathrm{mg} /$ day and two older children (10-11 years of age) up to $40 \mathrm{mg} /$ day. Prior to the inclusion into the study, an independent physician assessed the effectiveness of the medication.

In sum, we applied the following inclusion criteria: (a) diagnosis of ADHD combined subtype, (b) age between 7 and 12 years, (c) stable long-term medication (methylphenidate), and (d) an IQ > 80 as measured with the Kaufmann Assessment Battery for Children (K-ABC; Melchers and Preuß, 1991). Exclusion criteria were (a) maternal drug abuse in pregnancy, (b) premature birth ( $<32$ weeks) and low birth weight $(<2000 \mathrm{~g})$, (c) enrollment in special education settings, (d) neurological or chronic internal diseases, (e) Autism Spectrum, psychotic, bipolar, severe anxiety, and depressive disorder, and ( $\mathrm{f}$ ) any treatment with psychotropic drugs besides methylphenidate. The ethics review board of the Saarland Medical Association approved this training study. Written informed consent was given by one of the parents for all participating children. Subjects were paid 60 EUR for participating in the study.

Table 1 | Outline of the training protocol.

\begin{tabular}{lllll}
\hline Pretest session $\mathbf{1}$ & Training sessions 2-5 & Posttest 1 session $\mathbf{6}$ & Training sessions 7-10 & Posttest 2 session 11 \\
\hline BOTH GROUPS & GROUP 1 & BOTH GROUPS & GROUP 1 & BOTH GROUPS \\
Single-tasks (tasks A and B) & Single-task training & Single-tasks (tasks A and B) & Task-switching training & Single-tasks (tasks A and B) \\
Task-switching (tasks A and B) & (tasks C and D) & Task-switching (tasks A and B) & (tasks C and D) & Task-switching (tasks A and B) \\
COGNITIVE BATTERY & GROUP 2 & COGNITIVE BATTERY & GROUP 2 & COGNITIVE BATTERY \\
Stroop task & Task-switching training & Stroop task & Single-task training & Stroop task \\
Verbal working memory & (tasks C and D) & Verbal working memory & (tasks C and D) & Verbal working memory \\
Fluid intelligence & & Fluid intelligence & & Fluid intelligence \\
Control measures & & Control measures & & Control measures \\
Demographic questionnaire & & & &
\end{tabular}

Table 2 | Mean (SD) age, IQ, and sum scores on the FBB-HKS parent rating scale as a function of group at pretest.

\begin{tabular}{|c|c|c|c|c|}
\hline & \multicolumn{2}{|c|}{ Group 1 (single-task training first) } & \multicolumn{2}{|c|}{ Group 2 (task-switching training first) } \\
\hline & $M$ & SD & $M$ & SD \\
\hline IO & 107 & 14 & 103 & 11 \\
\hline FBB-HKS: severity of inattention & 13.8 & 4.6 & 14.4 & 7.7 \\
\hline FBB-HKS: severity of hyperactivity/impulsivity & 15.2 & 7.4 & 13.7 & 5.4 \\
\hline FBB-HKS: generalized hyperactivity/impulsivity problems & 9.9 & 5.0 & 9.0 & 5.5 \\
\hline
\end{tabular}

FBB-HKS scores are based on 20 items describing behavioral problems associated with ADHD and its subjective experienced severity. Parents were to rate the statements on a scale from 0 (not at all) to 3 (very much). Higher values correspond to more severe symptoms. 


\section{PROCEDURE}

For ethical reasons, all children in this study performed the training intervention (i.e., the task-switching training) but at different times during the training protocol. Therefore, transfer of taskswitching training was assessed by means of a pretest-trainingposttest 1-training-posttest 2 design (see Table 1 ). To determine the transfer scope, the pretest and posttest sessions consisted of a structurally similar, but new switching task and a battery of several cognitive tasks that are assumed to measure executive control as well as fluid intelligence. All training conditions included four sessions, each of them lasting about 30-40 min. The training protocol was carried out over a time period of 11 weeks, that is, the children performed approximately one session per week, similar to the training protocol of our previous training study (Karbach and Kray, 2009). Three expert experimenters (one psychologist and two research assistants) administered the tests and experimental tasks. They were randomly assigned to the test sessions.

\section{Pretest and posttest assessment}

Task-switching. We used the same task-switching paradigm as in one of our previous training studies (cf. Karbach and Kray, 2009). In this type of paradigm, the participants worked through single-task blocks (i.e., performing task A or B only) and through mixed-task blocks requiring the switching between both tasks A and B on every second trial. Participants received no task cues and had to keep track of the task sequence. In task A, participants were to decide whether a picture showed a fruit or a vegetable ("food" task), and to respond by pressing a left or right response key, respectively. In task B, they were to decide whether the picture was small or large ("size" task) and they also responded with a left or right response key. The same two response keys were used for both tasks and all stimuli were ambiguous. Stimuli consisted of 16 fruit and 16 vegetable pictures and each one of them was presented in a large and a small version.

Children first performed two single-task practice blocks (each consisting of 17 trials) and then worked through 20 experimental blocks ( 8 single-task and 12 mixed-task blocks, each consisting of 17 trials). The order of blocks was random with the constraint that two single and two mixed-task blocks were grouped together. At the beginning of each trial, a fixation cross appeared for $1400 \mathrm{~ms}$, followed by the target that was presented until the subject responded. After $25 \mathrm{~ms}$, the next fixation cross appeared. The children were instructed to respond as fast and as accurately as possible. After each block, subjects received feedback about their mean speed and accuracy of responding.

Cognitive test battery. The cognitive test battery included several experimental tasks and tests measuring executive control (inhibitory control, verbal working memory) and fluid intelligence. The pre- and post-test assessment took about 60-70 min.

We applied a modified version of the "Color-Stroop Task" (Stroop, 1935). In this version, children were shown words (e.g., "red," "tree") presented in red, blue, green, or yellow font successively on the computer screen. The color words were presented either in the congruent color or in an incongruent color. Children were to indicate the color of the words as quickly as possible by pressing one of four response buttons. Participants first performed two practice blocks (à 12 trials) and then four experimental blocks (à 24 trials). Stimuli were presented until the subject responded or for a maximum of $2000 \mathrm{~ms}$. The time window between the response and the next stimulus was $700 \mathrm{~ms}$. The Stroop interference effect was defined as the difference in mean performance between incongruent and congruent trials.

Verbal WM was assessed with the test "Digit Sorting" (cf. Kray and Lindenberger, 2000). In this test, the experimenter read aloud a series of digits ranging in value from 1 to 20 . The participants were to repeat the digits by sorting them in numerical order. The number of digits in each series varied between three and seven. Children first performed three practice series à three digits. The test started with three series à three digits, and then the number of digits per series was increased by one after each third series. The task was aborted after three consecutive erroneous responses. The test score was the number of correctly solved items.

We applied the matrix reasoning test from the German version of the Wechsler Intelligence Scale for Children (WISC-IV; Petermann and Petermann, 2007). In this test, the children were presented with a partially filled grid and asked to select the item that properly completed the matrix. Participants first completed three practice items, followed by up to 35 test items. The task was aborted after four consecutive erroneous responses or if four out of five consecutive items were not successfully completed. The test score was the number of correct responses.

In addition, we included two control measures on which we expected no positive transfer of the switching training. As a measure of perceptual speed of processing, we applied the DigitSymbol Substitution test (Wechsler, 1982). Children saw a template containing nine digit-symbol mappings on the top of the page. Below, they saw 100 digits without the corresponding symbols. They were instructed to fill in the correct symbols as fast as possible. The test score was the number of correctly completed symbols after $90 \mathrm{~s}$. As a measure of semantic knowledge, we used the Spot-a-Word test (Lehrl, 1977). In this test, 35 items are presented successively on the computer screen. Each item contains one correct word and four non-words. The participants were asked to find the one correct word. The test score was the number of correctly identified words.

The order of cognitive tasks and tests was constant during pre- and post-test assessment and were applied in the following order: Digit-Symbol Substitution Test, Task-Switching, Color-Stroop Task, Digit Sorting, Wechsler Intelligence Scale, and Spot-a-Word Test.

Training intervention. In the single-task training sessions, the children performed single-task blocks including either task C or task D. In the task-switching training sessions, the participants performed mixed-task blocks, that is, they were instructed to switch between both tasks $\mathrm{C}$ and $\mathrm{D}$ on every second trial. The experimental procedure during the training intervention was identical to the one applied at pretest and posttest except that children performed different tasks (tasks C and D). In task C ("transportation" task), subjects were to decide whether the pictures showed planes or cars, and in task D, ("number" task) whether one or two planes/cars were presented. They started with two 
practice blocks (à eight trials) followed by 24 experimental blocks (à 17 trials). In single-task training sessions, the children also started with two practice blocks (à eight trials) and then performed 24 experimental blocks (à 17 trials; 12 blocks of task C; and 12 blocks of task D in an alternating sequence). Overall all children worked through 1696 training trials in each training condition.

\section{DATA ANALYSIS}

Analyses for the switching and the Color-Stroop tasks were focused on mean RT for correct responses. We also analyzed response accuracy (\% errors) but consistent with previous data, we found no transfer of training (Karbach and Kray, 2009). Practice blocks and the first trial in each block were excluded from data analyses. For all remaining tasks of this study, the analyses were based on accuracy (number of correct responses). In order to test for between-group training effects, we run analyses of variance (ANOVA) with the between-subjects factor Group (group 1: single-task first, group 2: switching first). For the evaluation of transfer effects, we also calculated Cohen's $d$ (Cohen, 1977), or the standardized mean difference in performance between pretest and posttests (Verhaeghen et al., 1992). That is, the pretest-posttest differences (for each of the two groups) were divided by the pooled SD for test occasions. We then corrected all $d$ values for small sample bias using the Hedges and Olkin correction factor $\left(d^{\prime}\right.$; Hedges and Olkin, 1985).

\section{RESULTS}

\section{TRAINING EFFECTS}

To test for between-group differences in training-related benefits, we ran two ANOVAs, the first one for the single-task conditions and the second one for the task-switching conditions. Figures 1A,B show the latencies a function of Training Session (1-4) and experimental Group (group 1, group 2).

\section{Single-task training}

Training-related changes were analyzed with Group (group 1: single-task first, group 2: switching first) as between-subjects factor and Session (S1, S2, S3, S4) as within-subjects factor. Results showed a main effect of Session, $F(3,54)=5.65, p<0.01$, $\eta_{\mathrm{p}}^{2}=0.24$, with a quadratic slope, $F(1,54)=16.99, p<0.001$, $\eta_{\mathrm{p}}^{2}=0.49$, indicating that latencies increased from session 1 to sessions 2 and 3 but decreased again in session 4 (see Figure 1A). Neither the main effect of Group nor the interaction with Session was significant.

\section{Task-switching training}

The ANOVA including the between-subjects factor Group (group 1: single-task first, group 2: switching first) and the two withinsubjects factors Session (S1, S2, S3, S4) and Trial Type (non-switch, switch) showed a main effect of Session, $F(3,54)=5.05, p<0.01$, $\eta_{\mathrm{p}}^{2}=0.22$, indicating that latencies decreased as a function of training, and a main effect of Trial Type, $F(1,18)=23.84, p<0$. $001, \eta_{\mathrm{p}}^{2}=0.57$ (switching costs). An interaction between Session and Trial Type indicated that switching costs were reduced as a function of training, $F(3,18)=4.21, p=0.01, \eta_{\mathrm{p}}^{2}=0.19$ (see Figure 1B). Neither the main effect nor the interactions with the factor Group reached significance (all $p>0.46$ ).

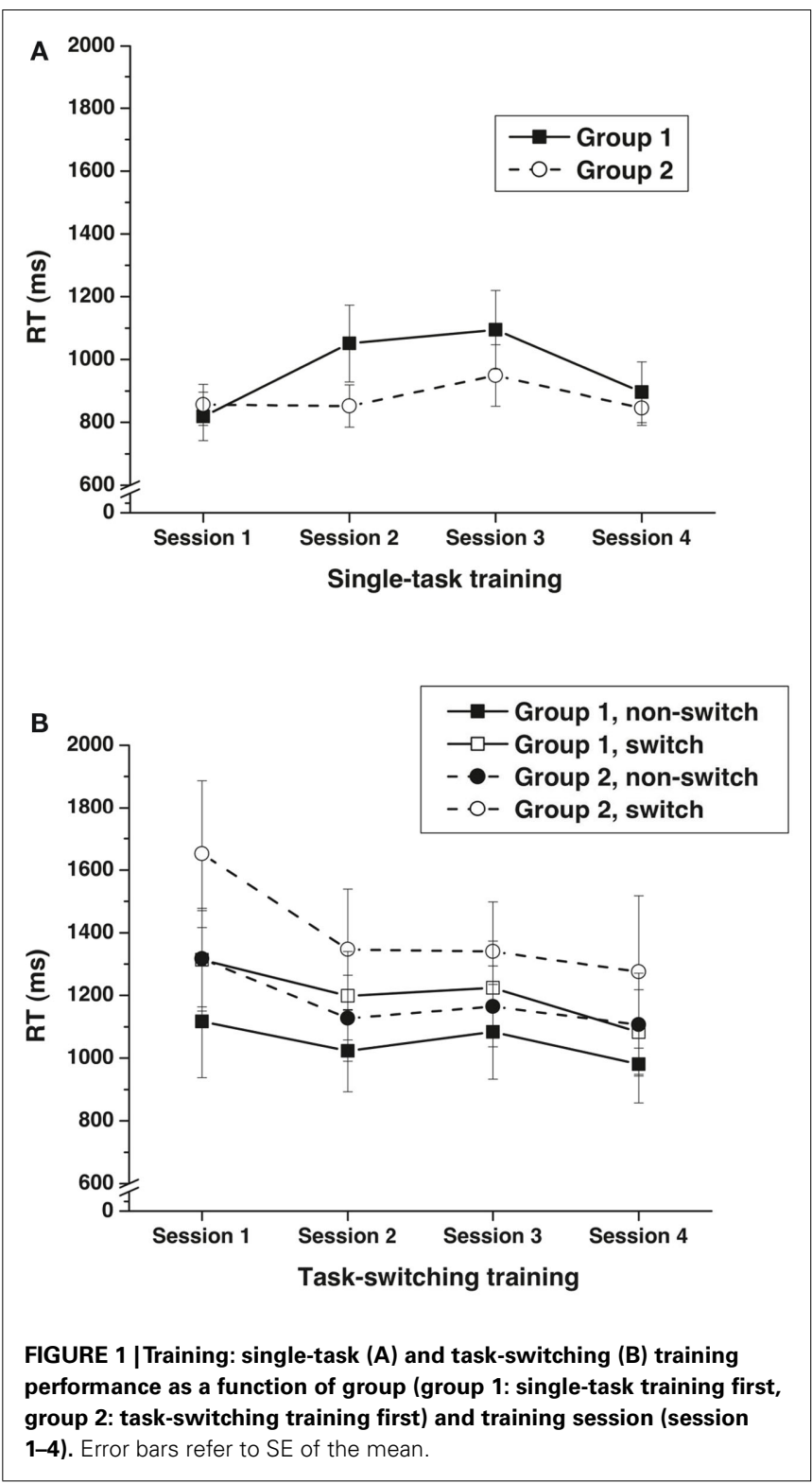

\section{ANALYSIS OF PRETEST DATA}

In order to make sure that transfer effects were not confounded with pre-existing differences in baseline performance, we tested for between-group differences at pretest before analyzing near and far transfer. ANOVAs with the between-subjects factor Group (group 1 , group 2) showed no significant group differences on any of the tasks (task-switching: $p=0.65$, interference control: $p=0.79$, verbal WM: $p=0.66$, fluid intelligence: $p=0.54$, perceptual speed: $p=0.82$, semantic knowledge: $p=0.65$ ).

\section{NEAR TRANSFER EFFECTS}

To investigate near transfer effects, we ran an ANOVA including the between-subjects factors Group (group 1: single-task first, group 2: switching first), and the within-subjects factors Trial Type (single, non-switch, switch), and Testing Time (pretest, posttest 1 , posttest 2). As in previous studies (e.g., Kray and Lindenberger, 
2000), mixing and switching costs were defined as two orthogonal contrasts. In the first contrast, the mean performance in single trials was tested against the mean performance on non-switch and switch trials (mixing costs), and in the second contrast mean performance on non-switch trials was tested against the mean performance on switch trials (switching costs). Training-specific effects were assessed by computing two contrasts for the factor Testing Time (pretest, posttest 1, posttest 2): The first contrast compared mean performance at pretest and posttest 1 , and the second one compared mean performance at posttest 1 and posttest 2. The means and SD of all experimental conditions are shown in Table 3. Mixing and switching costs as a function of testing time for both groups are displayed in Figures 2A,B.

The overall ANOVA showed a main effect of Trial Type, $F(2,36)=41.55, p<0.001, \eta_{\mathrm{p}}^{2}=0.70$, revealing significant mixing costs and switching costs $(F(1,18)=35.42, p<0.001$, $\eta_{\mathrm{p}}^{2}=0.66$, and $F(1,18)=68.38, p<0.001, \eta_{\mathrm{p}}^{2}=0.79$, respectively). In addition, we found interactions between Trial Type and Testing Time, $F(4,18)=6.49, p<0.001, \eta_{\mathrm{p}}^{2}=0.27$, and Trial Type, Testing Time, and Group, $F(4,18)=3.51, p=0.01$, $\eta_{\mathrm{p}}^{2}=0.16$. Mixing costs were reduced from pretest to posttest 1 , $F(1,18)=14.57, p<0.001, \eta_{\mathrm{p}}^{2}=0.45$. This reduction was somewhat larger for group 2 (task-switching training; $d^{\prime}=1.4$ ) than for group 1 (single-task training; $d^{\prime}=0.6$ ), $F(1,18)=3.40, p=0.08$, $\eta_{\mathrm{p}}^{2}=0.16$. Consistently, there also was a reduction of mixing costs from posttest 1 to posttest 2 in group 1 (task-switching training; $d^{\prime}=1.2$ ) but increased costs in group 2 (single-task training; $\left.d^{\prime}=-0.7\right), F(1,18)=6.64, p<0.05, \eta_{\mathrm{p}}^{2}=0.27$ (see Figure 2A).
Switching costs were also reduced from pretest to posttest $1, F(1,18)=21.97, p<0.001, \eta_{\mathrm{p}}^{2}=0.55$. Although this effect was larger for group 2 (task-switching training; $d^{\prime}=2.6$ ) than for group 1 (single-task training; $d^{\prime}=1.0$ ), the interaction with group failed to reach significance $(p=0.17)$. The contrast between posttest 1 and posttest 2 showed a reduction of switching costs from posttest 1 to posttest 2 in group 1 (task-switching training; $d^{\prime}=0.4$ ) but an increase in group 2 (single-task training; $\left.d^{\prime}=-1.0\right), F(1,18)=5.02, p<0.05, \eta_{\mathrm{p}}^{2}=0.22$ (see Figure 2B).

\section{FAR TRANSFER EFFECTS}

We used a similar ANOVA design to examine far transfer effects of the training intervention. We first report the results on far transfer to other executive control tasks, that is, to interference control and verbal WM, followed by the findings on fluid intelligence (reasoning), and finally to the two control measures, speed of processing, and semantic knowledge. Data of all far transfer measures are shown in Table 3.

\section{Interference control}

Data were submitted to a three-way ANOVA with the factors Group (group 1: single-task first, group 2: switching first), Testing Time (pretest, posttest 1, posttest 2), and Trial Type (congruent, incongruent). We found a main effect of Testing Time, $F(2,36)=5.68, p<0.01, \eta_{\mathrm{p}}^{2}=0.24$, indicating that the participants responded faster at posttest 1 than at pretest $(p<0.05)$. The main effect of Trial Type pointed to reliable interference costs $F(1,18)=13.69, p<0.01, \eta_{p}^{2}=0.43$, while the main effect

Table 3 | Mean performance (SD) for the near transfer (task-switching) and far transfer (inhibition, working memory, fluid intelligence) as a function of testing time (pretest, posttest 1, posttest 2) and group (group 1, group 2).

\begin{tabular}{|c|c|c|c|c|c|c|c|c|c|c|c|c|}
\hline & \multicolumn{6}{|c|}{ Group 1 (single-task training first) } & \multicolumn{6}{|c|}{ Group 2 (task-switching training first) } \\
\hline & \multicolumn{2}{|c|}{ Pretest } & \multicolumn{2}{|c|}{ Posttest 1} & \multicolumn{2}{|c|}{ Posttest 2} & \multicolumn{2}{|c|}{ Pretest } & \multicolumn{2}{|c|}{ Posttest 1} & \multicolumn{2}{|c|}{ Posttest 2} \\
\hline \multicolumn{13}{|c|}{ TASK-SWITCHING (NEAR TRANSFER) } \\
\hline Single trials & 1026 & 304 & 1106 & 414 & 1109 & 485 & 1080 & 272 & 1021 & 377 & 1027 & 397 \\
\hline Switch trials & 1607 & 594 & 1487 & 641 & 1271 & 514 & 1715 & 480 & 1161 & 380 & 1503 & 869 \\
\hline Mixing costs & 409 & 274 & 290 & 208 & 100 & 157 & 455 & 250 & 113 & 246 & 352 & 483 \\
\hline Switching costs & 346 & 185 & 184 & 186 & 125 & 227 & 360 & 161 & 55 & 150 & 248 & 247 \\
\hline \multicolumn{13}{|c|}{ STROOPTASK (FAR TRANSFER) } \\
\hline Congruent trials & 899 & 205 & 843 & 163 & 788 & 99 & 859 & 235 & 836 & 221 & 840 & 230 \\
\hline Incongruent trials & 952 & 219 & 900 & 186 & 825 & 127 & 939 & 214 & 823 & 170 & 836 & 182 \\
\hline \multicolumn{13}{|c|}{ FLUID INTELLIGENCE (FAR TRANSFER) } \\
\hline Fluid intelligence & 21.9 & 4.9 & 23.3 & 2.8 & 23.3 & 4.8 & 20.4 & 5.7 & 20.7 & 4.7 & 21.4 & 3.1 \\
\hline \multicolumn{13}{|c|}{ CONTROL MEASURES } \\
\hline Perceptual speed & 32.4 & 8.7 & 37.4 & 9.4 & 38.6 & 10.2 & 31.3 & 12.0 & 36.5 & 11.6 & 36.6 & 11.2 \\
\hline Semantic knowledge & 9.2 & 2.5 & 10.4 & 3.3 & 11.0 & 3.9 & 9.8 & 3.3 & 8.8 & 2.9 & 9.7 & 3.5 \\
\hline
\end{tabular}




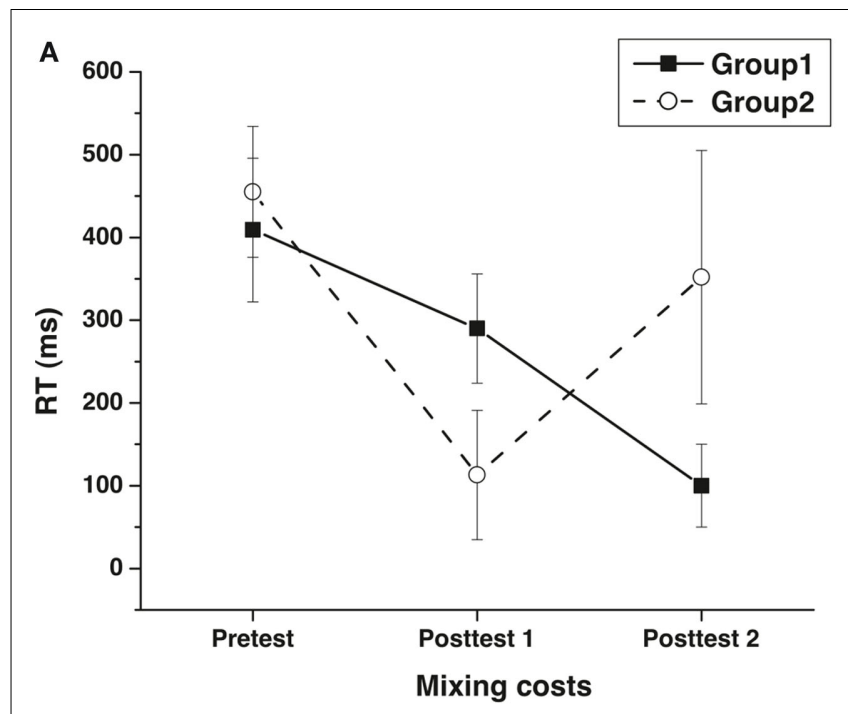

B

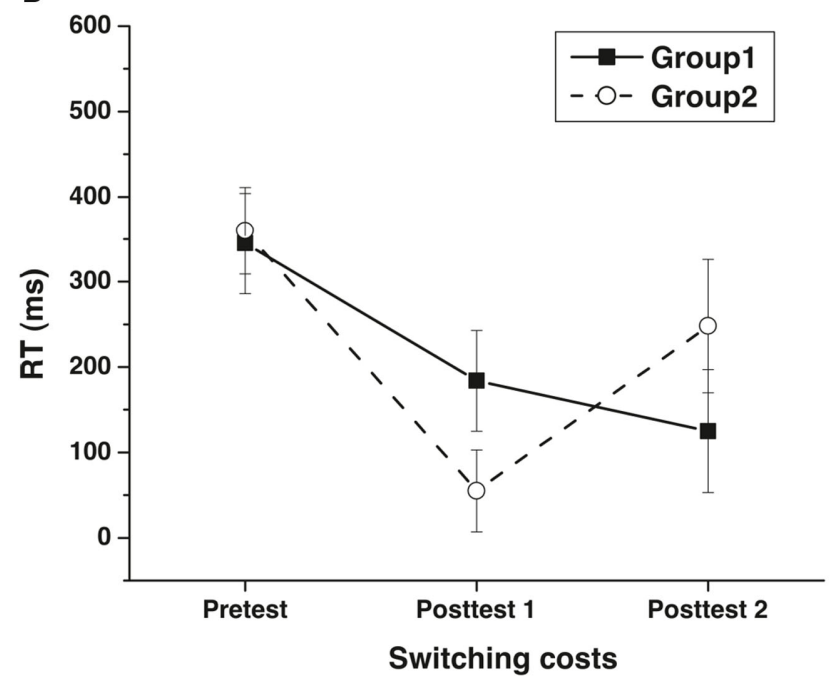

FIGURE 2 | Near transfer: mixing costs (A) and switching costs (B) as a function of group (group 1: single-task training first, group 2: task-switching training first) and testing time (pretest, posttest 1, posttest 2). Error bars refer to SE of the mean.

of Group failed to reach significance $(p=0.88)$. An interaction between Testing Time and Trial Type, $F(2,36)=3.80, p<0.05$, $\eta_{\mathrm{p}}^{2}=0.17$, revealed that interference costs were reduced from pretest to posttest $1, F(1,18)=7.07, p<0.05, \eta_{p}^{2}=0.28$. Importantly, we also found a marginally significant interaction between Testing Time, Trial Type, and Group, $F(2,36)=3.12, p=0.06$, $\eta_{\mathrm{p}}^{2}=0.15$. The contrast between pretest and posttest 1 showed that the reduction of interference costs was larger in group 2 (taskswitching training; $d^{\prime}=1.6$ ) than in group 1 (single-task training; $\left.d^{\prime}=0.1\right), F(1,18)=8.33, p=0.01, \eta_{\mathrm{p}}^{2}=0.32$ (see Figure 3A), but we obtained no larger reduction of interference costs in group 1 (task-switching training; $d^{\prime}=0.4$ ) than in group 2 (single-task training; $d^{\prime}=0.2$ ) from posttest 1 to posttest $2(p=0.58)$.
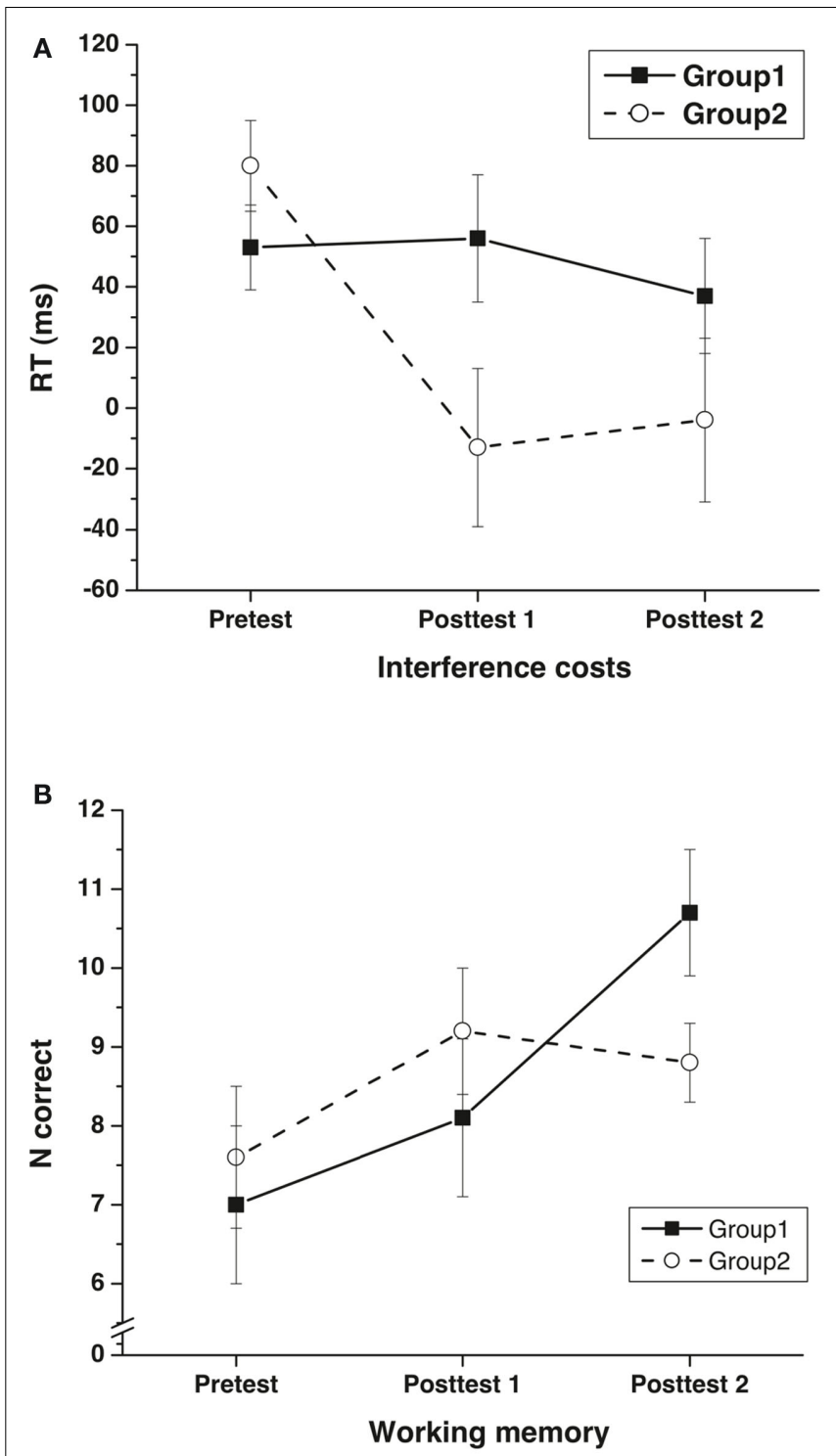

FIGURE 3 | Far transfer: interference costs (A) and working memory performance (B) as a function of group (group 1: single-task training first, group 2: task-switching training first) and testing time (pretest, posttest 1, posttest 2). Error bars refer to SE of the mean.

\section{Verbal working memory}

The ANOVA with the factors Group and Testing Time revealed a main effect of Testing Time, $F(2,36)=19.62, p<0.001, \eta_{p}^{2}=$ 0.52 , indicating that WM performance improved from pretest to posttest 1 and also from posttest 1 to posttest 2 (both $p s<0.01$ ). The main effect of Group was not significant $(p=0.95)$. An interaction between Group and Testing Time, $F(2,36)=8.41$, $p<0.001, \eta_{\mathrm{p}}^{2}=0.32$, showed larger performance improvements from posttest 1 to posttest 2 in group 1 (task-switching training; $d^{\prime}=0.9$ ) than in group 2 (single-task training; $d^{\prime}=-0.2$; see Figure 3B). However, no training-specific improvements were found from pretest to posttest 1 (single-task training: $d^{\prime}=0.3$; task-switching training: $\left.d^{\prime}=0.6 ; p=0.44\right)$. 


\section{Fluid intelligence (reasoning)}

The ANOVA with the factors Group and Testing Time neither revealed significant main effects nor an interaction (all $p s>0.26$ ).

\section{Control variables}

The ANOVA based for perceptual speed of processing showed a main effect of Testing Time, $F(2,36)=15.61, p<0.001, \eta_{\mathrm{p}}^{2}=$ 0.46 , with performance improvements from pretest to posttest 1 $(p<0.001)$, but neither the main effect for Group nor the interaction with Testing Time reached significance (both $p s>0.77$ ). The analysis of the semantic knowledge task showed no significant effects (all $p s>0.31$ ).

\section{DISCUSSION}

Children with ADHD showed a reduction of switching costs throughout the task-switching training, suggesting that they already benefited from a relatively short intervention of four training sessions. Even more important than the training-related improvements in switching performance are the near and far transfer effects observed in this study. As illustrated in Figure 2A, the task-switching training led to a substantial reduction of mixing costs in a similar switching task with similar effect sizes for the two groups (goup1: $d^{\prime}=1.4$; group $2: d^{\prime}=1.2$ ), which can be considered as large effects (Verhaeghen et al., 1992). Interestingly, the treatment effect was about the same independently of whether the subjects had already performed the single-task training or not. In contrast, the task-switching training resulted in a large reduction of switching costs in the group that performed the task-switching training first (group 2: $d^{\prime}=2.6$ ), but the reduction in the group that had already performed single-task training was only very small (group $1: d^{\prime}=0.4$ ), probably because there was not much room for improvement in task-switching (see Figure 2B). A similar pattern of findings occurs for the training-related changes in inhibitory control. While the group that performed the taskswitching training first showed a substantial reduction of interference costs (group 2: $d^{\prime}=1.6$ ), this reduction was, however, only of small size for the group that had already performed the singletask training (group 1: $d^{\prime}=0.4$ ). We obtained a large increase in verbal WM in the group that performed the task-switching training first (group 2: $d^{\prime}=0.9$ ) while the effect size was only medium for the group that had already performed the single-task training (group 1: $\left.d^{\prime}=0.6\right)$. In contrast to our previous study with young children (Karbach and Kray, 2009), we did not find transfer of task-switching training to performance on a fluid intelligence test in children suffering from ADHD. However, it should be noted that we used different tests in both studies, which might explain the difference in findings.

In sum, the present study provided the first evidence for near and far transfer of task-switching training in children suffering from ADHD. It therefore is of major interest to examine whether the training was as effective in children with ADHD as it has previously been in healthy children. Comparing the results from the present study with our previous one (Karbach and Kray, 2009) showed that the effect sizes for the near transfer of taskswitching were higher in healthy children than in the ADHD sample in terms of mixing costs (mean $d^{\prime}$ healthy group $=2.1$, mean $d^{\prime}$ ADHD group $=1.3$ ) but similar in terms of switching costs (mean $d^{\prime}$ healthy group $=1.2$, mean $\mathrm{d}^{\prime}{ }_{\mathrm{ADHD}}$ group $\left.=1.5\right)$. Regarding the far transfer to interference control, we even found slightly higher effects sizes in the ADHD group than in the healthy sample (mean $d^{\prime}$ healthy group $=0.5$, mean $d^{\prime}$ ADHD group $=0.8$ ), while the transfer to WM was comparable across studies (mean $d^{\prime}$ healthy group $=0.9$, mean $d^{\prime}$ ADHD group $=0.8$ ). Thus, the general pattern of results across both groups showed the typical finding of larger effect sizes on near compared to far transfer tasks. In addition, the size of these effects was similar (with the exception of mixing costs), indicating that results of the ADHD children seem to be within the range of what has been reported for healthy children. Although this finding has to be replicated within a single study, it points to the potential for the application of relatively short cognitive interventions in clinically relevant populations.

Although there was evidence for training-specific improvements of the task-switching intervention, it should be noted that we also obtained transfer effects of medium sizes after the singletask training. One possible explanation of this finding is that ADHD children have major deficits in the control of attention and interference. Given that the stimuli in this study were ambiguous, even the single-task training may have resulted in a certain amount of training in executive control. This means that although the ADHD children were not trained in task-switching, they may have been trained in focusing their attention on relevant information while ignoring irrelevant task features.

Although we found large effect sizes for near and far transfer of task-switching training, this study has some limitations. First, the sample was relatively small so that some interactions of the expected training-specific effects were only marginally significant. Second, the fact that we only investigated male children limits the generalizability of our findings. Third, given our training design, we also observed a decrease in task-switching performance between the posttest 1 and posttest 2 for the group that performed the single-task training after the task-switching training (group 2), as illustrated in Figures 2A,B. One possible explanation for this finding is that the ADHD children suffered from a loss of motivation across the four easier single-task training sessions and were therefore also less motivated to perform the switching tasks at posttest 2. Another explanation would be that the decrease in performance reflects negative transfer in the sense that the intensive training in performing single-tasks interferes with the coordination of control processes required for the switching tasks. Unclear is, however, why this negative effect does not occur for group 1. Either way future research is needed to clarify the nature of this carryover effect. If training order effects influence motivation, future studies could additionally control for individual differences in motivation and self-regulatory strategies such as self-efficacy or active engagement in the training. Such individual characteristics have recently been found to moderate memory training and transfer effects in elderly subjects (e.g., West and Hastings, 2011). As children with ADHD have impairments in regulating and maintaining engagement in an activity for a longer period of time, these motivational factors might also contribute to differential training and transfer effects in this clinical group.

The present training study extended our knowledge regarding useful cognitive training interventions for children with combined 
ADHD who were on stable methylphenidate medication. Previous studies found that executive control functioning as well as academic skills and behavioral deficits can be improved by WM training and attentional control training (Kerns et al., 1999; Klingberg et al., 2002, 2005; Shalev et al., 2007). The intensity of the training was quite high in these studies [e.g., at least 25 training sessions in the Klingberg et al. (2005) study]. Results of our study suggest that performance improvements in executive control functioning can be achieved after a relatively short training intervention of four sessions in task-switching. However, whether even larger training effects can be achieved with adaptive or more intensive training procedures (Klingberg et al., 2002, 2005) and whether training

\section{REFERENCES}

American Psychiatric Association. (1994). Diagnostic and Statistical Manual of Mental Disorders DSM$I V$. Washington, DC: American Psychiatric Association.

Barkley, R. A. (1997). Behavioral inhibition, sustained attention, and executive functions: constructing a unifying theory of ADHD. Psychol. Bull. 121, 65-94.

Cepeda, N. J., Cepeda, M. L., and Kramer, A. F. (2000). Task switching and attention deficit hyperactivity disorder. J. Abnorm. Child Psychol. 28, 213-226.

Cepeda, N. J., Kramer, A. F., and Gonzalez de Sather, J. C. (2001). Changes in executive control across the life span: examination of task-switching performance. Dev. Psychol. 37, 715-730.

Cohen, J. (1977). Statistical Power Analysis for the Behavioral Sciences. New York: Academic Press.

Döpfner, M., and Görtz-Dorten, A. (2008). Diagnostik-System für Psychische Störungen im Kindes- und Jugendalter nach ICD-10 und DSMIV (DISYPS-II). Bern: Huber.

Froehlich, T. E., Lanphear, B. P., Epstein, J. N., Barbaresi, W. J., Katusic, S. K., and Kahn, R. S. (2007). Prevalence, recognition, and treatment of attention-deficit/hyperactivity disorder in a national sample of US children. Arch. Pediatr. Adolesc. Med. 161, 857-864.

Hedges, L. V., and Olkin, I. (1985). Statistical Methods for Meta-Analysis. Orlando: Academic Press.

Karbach, J., and Kray, J. (2009). How useful is executive control training? Age differences in near and far transfer of task-switching training. Dev. Sci. 12, 978-990.

Karbach, J., Mang, S., and Kray, J. (2010). Transfer of verbal self-instruction training in older age: evidence from task switching. Psychol. Aging 25, 677-683.

Kerns, K. A., Eso, K., and Thomson, J. (1999). Investigation of a direct intervention for improving attention in young children with ADHD. Dev. Neuropsychol. 16, 273-295.

Kiesel, A., Steinhauer, M., Wendt, M., Falkenstein, M., Jost, K., Philipp, A. M., and Koch, I. (2010). Control and interference in task switching - a review. Psychol. Bull. 136, 849-874.

Klingberg, T., Fernell, E., Olesen, P., Johnson, M., Gustafsson, P., Dahlström, K., Gillberg, C. G., Forssberg, H., and Westerberg, H. (2005). Computerized training of working memory in children with ADHD - a randomized, controlled trial. J. Am. Acad. Child Adolesc. Psychiatry 44, 77-186.

Klingberg, T., Forssberg, H., and Westeberg, H. (2002). Training of working memory in children with ADHD. J. Clin. Exp. Neuropsychol. 24, 781-791.

Kramer, A. F., Cepeda, M. L., and Cepeda, M. L. (2001). Methylphenidat effects on task-switching performance in attention-deficit/hyperactivity disorder. J. Am. Acad. Child Adolesc. Psychiatry 40, 1277-1284.

Kray, J., Eber, J., and Karbach, J. (2008). Verbal self-instructions in task-switching: a compensatory tool for action-control deficits in childhood and old age? Dev. Sci. 11, 223-236.

Kray, J., and Lindenberger, U. (2000). Adult age differences in task switching. Psychol. Aging 15, 126-147.

Lehrl, S. (1977). MWT-B: MehrfachWahl-Wortschatz-Test B. Erlangen: Straube.

effects can be maintained over a longer period of time has to be clarified in future studies. Another interesting question for future research with important clinical implications is to directly compare the effectiveness of the already existing training programs or to combine them in order to achieve an optimal cognitive intervention for children with ADHD.

\section{ACKNOWLEDGMENTS}

This research was funded by the German Research Foundation (DFG) through a grant to Jutta Kray (KR 1884/3-4). Special thanks go to Wera Otto and Cathia Loor for their help running the experiments.

Lövdén, M., Bäckman, L., Lindenberger, U., Scheafer, S., and Schmiedek, F. (2010). A theoretical framework for the study of adult cognitive plasticity. Psychol. Bull. 136, 659-676.

Melchers, P., and Preuß, U. (1991) K-ABC: Kaufman Assessment Battery for Children. Frankfurt: Swets \& Zeitlinger.

Minear, M., and Shah, P. (2008). Training and transfer effects in task switching. Mem. Cognit. 36, 1470-1483.

Miyake, A., Friedman, N. P, Emersom, M. J, Witzki, A. H, Howerter, A., and Wagner, T. D. (2000). The unity and diversity of executive functions and their contributions to complex "frontal lobe" tasks: a latent variable analysis. Cogn. Psychol. 41, 49-100.

Petermann, F., and Petermann, U. (2007). WISC-IV: Wechsler Intelligence Scale for Children - IV. Bern: Huber.

Rasmussen, P., and Gillberg, C. (2001). Natural outcome of ADHD with developmental coordination disorder at age 22 years: a controlled, longitudinal, community-based study. J. Am. Acad. Child Adolesc. Psychiatry 39, 1424-1431.

Shalev, L., Tsal, Y., and Mevorach, C. (2007). Computerized progressive attentional training (CPAT): effective direct intervention for children with ADHD. Child Neuropsychol. 13, 382-388.

Stroop, J. R. (1935). Studies of interference in serial verbal reactions. J. Exp. Psychol. 18, 643-662.

Unnewehr, S., Schneider, S., and Margraf, J. (1998). Kinder-DIPS. Diagnostisches Interview bei psychischen Störungen im Kindes und Jugendalter. Heidelberg: Springer.
Verhaeghen, P., Marcoen, A., and Goossens, L. (1992). Improving memory performance in the aged through mnemonic training: a metaanalytic study. Psychol. Aging 7, 242-251.

Wechsler, W. (1982). Manual for the Hamburg-Wechsler Intelligence Test for Adults (HAWIE). Bern: Huber.

West, R. L., and Hastings, E. C. (2011). Self-regulation and recall: growth curve modeling of intervention outcomes for older adults. Psychol. Aging 26, 803-812.

Willcutt, E. G., Doyle, A. E., Nigg, J. T, Faraone, S. V., and Pennington, B. F. (2005). Validity of the executive function theory of attention deficit/hyperactivity disorder: a metaanalytic review. Biol. Psychiatry 57, 1336-1346.

Conflict of Interest Statement: The authors declare that the research was conducted in the absence of any commercial or financial relationships that could be construed as a potential conflict of interest.

Received: 28 September 2011; accepted: 16 December 2011; published online: 03 January 2012.

Citation: Kray J, Karbach J, Haenig $S$ and Freitag C (2012) Can taskswitching training enhance executive control functioning in children with attention deficit/-hyperactivity disorder? Front. Hum. Neurosci. 5:180. doi: 10.3389/fnhum.2011.00180

Copyright $(2012$ Kray, Karbach, Haenig and Freitag. This is an open-access article distributed under the terms of the Creative Commons Attribution Non Commercial License, which permits noncommercial use, distribution, and reproduction in other forums, provided the original authors and source are credited. 